\title{
Comparison of Models for Heat Transfer in High-Density Fibrous Insulation
}

\author{
Sergio A. Carvajal ${ }^{1}$, Edward J. Garboczi ${ }^{2}$, and Robert R. Zarr ${ }^{3}$ \\ ${ }^{1}$ Instituto Nacional de Metrología de Colombia \\ Bogotá, DC 111321 Colombia \\ ${ }^{2}$ National Institute of Standards and Technology \\ Boulder, CO 80305 USA \\ ${ }^{3}$ National Institute of Standards and Technology \\ Gaithersburg, MD 20899 USA \\ sacarvajal@inm.gov.co \\ edward.garboczi@nist.gov \\ robert.zarr@nist.gov
}

\begin{abstract}
This study evaluated different models for calculating the effective thermal conductivity of fibrous insulation by comparing predicted values with certified values of Standard Reference Material 1450c, Fibrous Glass Board. This comparison involved the coupled effects of radiation and conduction heat transfer. To support these comparisons, the fiber diameter distribution was measured using X-ray computed tomography, and this distribution was used in several heat transfer models considered in this paper. For the evaluation of the radiative heat transfer, the diffusion approximation, the Schuster-Schwarzschild approximation, and the Milne-Eddington approximation were considered. The conduction of the gas and the fibers was treated by the kinetic theory and a semi-empirical model, respectively. Two models were considered for the evaluation of the radiative properties: the large specular reflecting approach and the application of Mie theory for media composed of infinite cylinders.
\end{abstract}

Key words: conduction; fiber, insulation; models; radiation heat transfer; SRM 1450c; X-ray computed tomography.

Accepted: February 19, 2019

Published: May 13, 2019

https://doi.org/10.6028/jres.124.010

\section{Glossary}

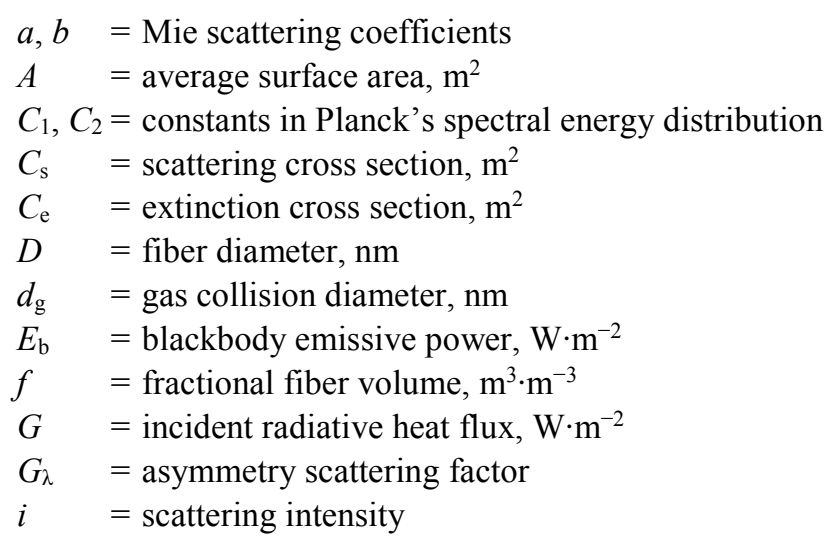


$\mathrm{Kn} \quad=$ Knudsen number

$k=$ thermal conductivity, $\mathrm{W} \cdot \mathrm{m}^{-1} \cdot \mathrm{K}^{-1}$

$k_{\text {app }}=$ apparent thermal conductivity, $\mathrm{W} \cdot \mathrm{m}^{-1} \cdot \mathrm{K}^{-1}$

$k_{0} \quad=$ wavenumber in the outer medium, $\mathrm{m}^{-1}$

$k_{\mathrm{B}} \quad=$ Boltzmann constant, $\mathrm{m}^{2} \cdot \mathrm{kg} \cdot \mathrm{s}^{-2} \cdot \mathrm{K}^{-1}$

$L \quad=$ specimen length, $\mathrm{m}$ (see Fig. 1)

$L_{\mathrm{c}} \quad=$ characteristic length for fibers perpendicular to the heat flow, $\mathrm{m}$

$L_{\mathrm{f}} \quad=$ length of fibers, $\mathrm{m}$

$m \quad=$ solid conduction exponent term

$N \quad=$ number of fibers per volume

$N_{\mathrm{f}} \quad=$ distribution of the fiber size

$P \quad=$ gas pressure, $\mathrm{Pa}$

$\operatorname{Pr} \quad=$ Prandtl number

$Q_{\text {abs }}=$ absorption efficiency

$q_{\mathrm{R}} \quad=$ radiative heat flux, $\mathrm{W} \cdot \mathrm{m}^{-2}$

$r \quad=$ radial coordinate

$\operatorname{Re}=$ real part of a complex number

$T=$ temperature, $\mathrm{K}$

$T_{i, j} \quad=$ elements of the $T$-matrix

$x=$ dimensional coordinate, $\mathrm{m}$

$y \quad=$ dimensional coordinate, $\mathrm{m}$

$x_{j} \quad=$ fraction of fibers of radius $r_{j}$

$p \quad=$ phase scattering function

$\alpha=$ absorptivity

$\beta \quad=$ extinction coefficient, $\mathrm{m}^{-1}$

$\gamma \quad=$ specific heat ratio for standard air

$\varepsilon \quad=$ emissivity

$\zeta \quad=$ thermal accommodation coefficient

$\eta \quad=$ scattering angle

$\theta \quad=$ observation angle

$\kappa=$ absorption coefficient, $\mathrm{m}^{-1}$

$\lambda=$ wavelength, $\mathrm{nm}$

$\lambda_{\mathrm{m}} \quad=$ molecular mean free path, $\mathrm{nm}$

$\mu \quad=$ direction cosine of polar angle

$\rho \quad=$ bulk density, $\mathrm{kg} \cdot \mathrm{m}^{-3}$

$\sigma \quad=$ Stefan-Boltzmann constant, $\mathrm{W} \cdot \mathrm{m}^{-2} \cdot \mathrm{K}^{-4}$

$\sigma_{\mathrm{s}} \quad=$ scattering coefficient, $\mathrm{m}^{-1}$

$\varphi \quad=$ incident angle

$\Phi, \Psi=$ parameters for gas thermal conductivity

$\omega=$ azimuthal angle

$\omega_{0} \quad=$ albedo of scattering

\section{$\underline{\text { Additional subscripts and superscripts }}$}

$L \quad$ denotes at $x=L$

0 denotes at $x=0$

+ denotes from right

- denotes from left

$f$ denotes fiber 


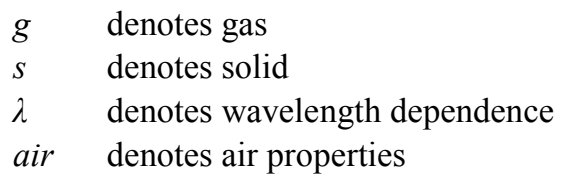

\section{Introduction}

The purpose of this study was to evaluate several heat transfer models for predicting the effective thermal conductivity of a high-density fibrous insulation $\left(155.5 \mathrm{~kg} \cdot \mathrm{m}^{-3}\right)$ from $280 \mathrm{~K}$ to $340 \mathrm{~K}$ at atmospheric pressures in air. An understanding of the physics of thermal conductivity is important in the design and improvement of associated measurement techniques.

The modelling of heat transfer through fibrous media is especially challenging due to the complexity associated with the formulation of the coupled phenomena of radiation and conduction. A large body of literature has been produced on this topic. In general, the differences in the methods proposed are based on the estimate of the individual-phase radiative properties and on the analysis of radiative heat transfer in the fibrous media. Excellent reviews are given by Lee et al. [1] and Stephenson [2]. Due to its simplicity, the most-used model for radiation heat transfer is the diffusion approximation. Zhang et al. [3] and Daryabeigi [4], however, have modeled the radiation heat transfer using modified two-flux approximations.

To solve the radiative heat transfer equation, the optical properties of the fibers must be determined. Due to the complexity associated with radiation scattering and absorption in relevant media, a common practice is to estimate the unknown parameters from the solution of the heat transfer equation $[2,5,6]$. On the other hand, previous works of Tong and Tien [7] and Lee [8-11] proposed a rigorous formulation of the Maxwell equations to account for the main properties of the media: fiber orientation, fiber particle size (i.e., diameter) distribution, and dependent scattering, which occurs when the scattering by one particle is affected by the presence of neighboring particles.

For gas conduction, almost all researchers use the kinetic theory to model the phenomena [12]. Semi-empirical models have been proposed for solid fiber conduction. Some of these models require measurement at vacuum and cryogenic conditions to evaluate unknown parameters [13], and other theoretical models are based on Fricke's method for electrical conductivity [14].

\section{Heat Transfer Models}

The main mechanisms involved in heat transfer through fibrous insulation are conduction through the gas and solid fiber, thermal radiation, and natural convection. However, for still air, natural convection can be assumed to be negligible for densities greater than $20 \mathrm{~kg} \cdot \mathrm{m}^{-3}$, because the fibers partition the gas into sufficiently small pores [14, 15] so that natural convection is negligible.

In the general form, the energy balance of a body between two parallel isothermal plates (Fig. 1) that are at different temperatures, at steady state, can be expressed as [16]

$$
\frac{d}{d x}\left(k(T) \frac{d T}{d x}\right)-\frac{d q_{R}}{d x}=0
$$

where edge effects are ignored to allow only a one-dimensional equation, and

$$
T(0)=T_{0}
$$

and

$$
T(L)=T_{L}
$$


Here, $k$ is the thermal conductivity, $T$ is temperature, $x$ is the dimensional coordinate parallel to the heat flow, $q_{\mathrm{R}}$ is the radiative heat flux, $L$ is the length of the body, and $T_{0}$ and $T_{L}$ are the temperatures in the cold and the hot plates, respectively.

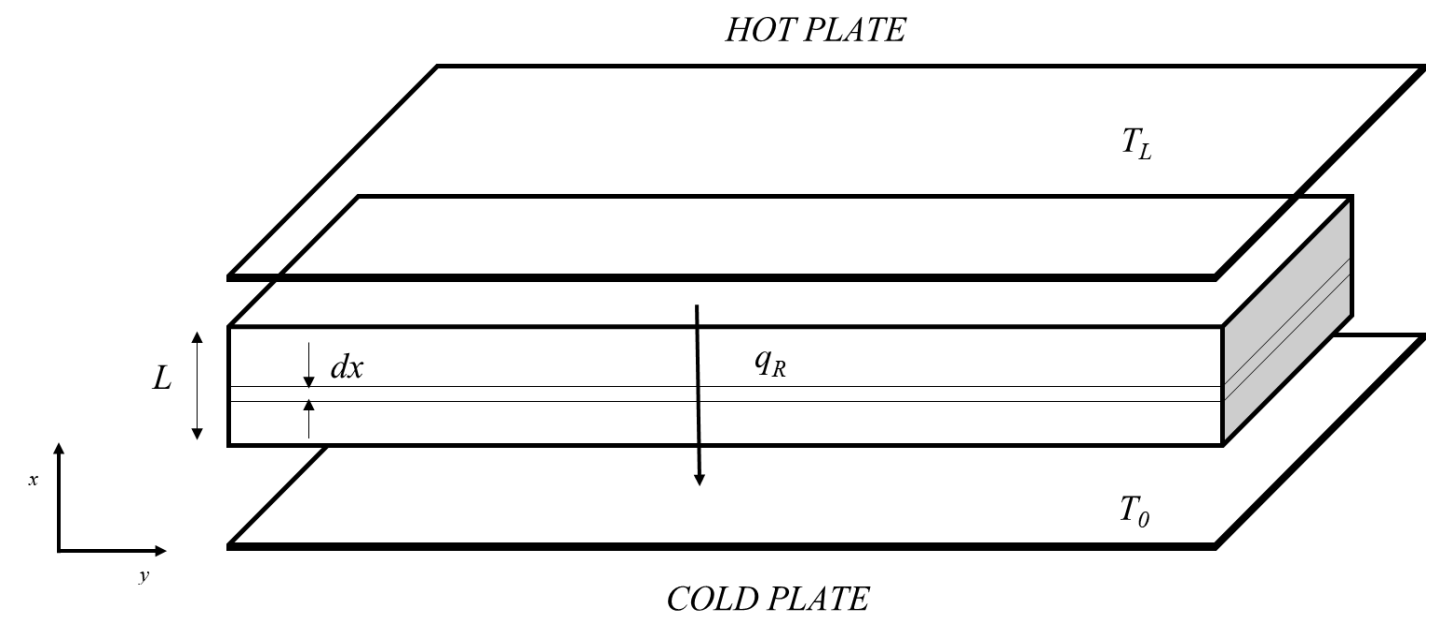

Fig. 1. Heat flow between isothermal plates.

Heat conduction through the gas and solid fibers is well described by application of the Fourier law [15] for modeling the interaction of the gas and solid conductivities. The main differences in the models come from the evaluation of the radiation term. In this paper, the radiative heat transfer will be evaluated by the following three approximations: the diffusion approximation, the Schuster-Schwarzschild approximation, and the Milne-Eddington approximation.

\subsection{Radiative Transfer Equations (RTEs)}

\subsubsection{Diffusion Approximation}

The first approach incorporates the Rosseland diffusion approximation [17] for an optically dense medium, where the radiative heat flux is given by:

$$
q_{R}=-\frac{16 \sigma T^{3}}{3 \beta} \frac{d T}{d x}
$$

where $\sigma$ is the Stefan-Boltzmann constant, and $\beta$ is the extinction coefficient. Substituting Eq. (3) in Eq. (1) yields:

$$
\frac{d}{d x}\left[\left(\frac{16 \sigma T^{3}}{3 \beta}+k\right) \frac{d T}{d x}\right]=0 .
$$

\subsubsection{Schuster and Schwarzschild Approximation}

The model proposed by Farnworth [18] and evaluated by Du et al. [19] and Mavromatidis et al. [20] is based on the two-flux approach by Schuster and Schwarzschild for radiative flux and negligible scattering of radiation by the fibers. The radiative heat transfer can be expressed as

$$
q_{R}=G^{+}-G^{-}
$$




$$
\begin{aligned}
& \frac{d G^{+}}{d x}=-\beta G^{+}+\beta \sigma T^{4}, \\
& \frac{d G^{-}}{d x}=\beta G^{-}-\beta \sigma T^{4},
\end{aligned}
$$

where $G^{+}$and $G^{-}$represents the incident radiative heat flux from below and above, respectively, and the boundary conditions are

$$
\begin{gathered}
\left(1-\varepsilon_{1}\right) G^{-}(0)+\varepsilon_{1} \sigma T_{0}^{4}=G^{+}(0), \\
\left(1-\varepsilon_{2}\right) G^{+}(L)+\varepsilon_{2} \sigma T_{L}^{4}=G^{-}(L),
\end{gathered}
$$

and $\varepsilon_{1}$ and $\varepsilon_{2}$ are the emissivities of the surfaces at $x=0$ and $x=L$, respectively. Substituting Eqs. (5) through (7) in Eq. (1) and simplifying yields:

$$
k \frac{d^{2} T}{d x^{2}}+\beta\left(G^{+}+G^{-}\right)-2 \beta \sigma T^{4}=0 .
$$

Equations (6), (7), and (10) were solved simultaneously by iteration as follows:

- For the first iteration, assume a linear temperature profile.

- Assume the magnitude of the incident radiative heat flux $G^{-}(0)$ arbitrarily and calculate $G^{+}(0)$ from Eq. (8). Calculate $G^{+}(x)$ from Eq. (6) using backward finite difference.

- $\quad$ Calculate $G^{-}(L)$ from Eq. (9) with the $G^{+}(L)$ calculated in the previous step.

- $\quad$ From Eq. (7), calculate $G^{-}(x)$ using forward finite difference.

- Calculate the (new) temperature profile with Eq. (10) and repeat the above steps. Mavromatidis et al. [20] showed that the model provides good agreement in multilayer thermal insulators.

\subsubsection{Milne-Eddington Approximation}

The radiative heat flux is calculated by assuming that the media behaves as a gray body (i.e., the radiative properties are independent of the wavelength) and using the first and second moments of the radiative transfer equation. Equations (11) through (13) represent the resulting model [16]:

$$
\begin{gathered}
\frac{d q_{R}}{d x}=\beta\left(1-\omega_{0}\right)\left(4 \sigma T^{4}-G\right), \\
\frac{d G}{d x}=-3 \beta q_{R} \\
-\frac{1}{3 \beta^{2}\left(1-\omega_{0}\right)} \frac{d^{2} G}{d x^{2}}+G=4 \sigma T^{4},
\end{gathered}
$$

where $G$ is the incident radiative heat flux, and $\omega_{0}$ is the albedo scattering. Daryabeigi et al. [21] used this model for the optimal design of multilayer insulation subjected to reentry aerodynamics heating. Zhang et al. [3] compared the model with experimental data at high temperatures under vacuum conditions and obtained agreement of $13.5 \%$. 


\section{Journal of Research of the National Institute of Standards and Technology}

\subsection{Gas Thermal Conductivity}

For the gaseous thermal conductivity, the model used by Daryabeigi [4] was evaluated

$$
k_{g}=\frac{k_{a i r}}{\Phi+2 \Psi\left(\frac{2-\zeta_{g}}{\zeta_{g}}\right)\left(\frac{2 \gamma}{\gamma+1}\right)\left(\frac{1}{\operatorname{Pr}}\right)\left(\frac{\lambda_{m}}{L_{c}}\right)},
$$

where $k_{\mathrm{g}}$ is the thermal conductivity in the gas phase, $k_{\text {air }}$ is the thermal conductivity of air at atmospheric pressure, $\zeta_{\mathrm{g}}$ is the thermal accommodation coefficient (which is a measure of the thermal energy transfer between a gas molecule and the surface), $\gamma$ is the ratio of the heat capacity at constant pressure to the heat capacity at constant volume of the air, and $\operatorname{Pr}$ is the Prandtl number. Values for $\Phi$ and $\Psi$ are related to the Knudsen number (Kn) as summarized in Table 1 [4].

Table 1. Parameters of gas thermal conductivity.

\begin{tabular}{ccc}
\hline $\mathrm{Kn}\left(\frac{\lambda_{m}}{L_{c}}\right)$ & $\Phi$ & $\Psi$ \\
\hline$<0.01$ & 1 & 0 \\
\hline $0.01-10$ & 1 & 1 \\
\hline$>10$ & 0 & 1 \\
\hline
\end{tabular}

The molecular mean free path $\lambda_{\mathrm{m}}$ is given by

$$
\lambda_{\mathrm{m}}=\frac{k_{B} T}{\sqrt{2} \pi d_{g}^{2} P}
$$

where $k_{B}$ is the Boltzmann constant, $d_{\mathrm{g}}$ is the gas collision diameter, and $P$ is the pressure. The characteristic length $L_{\mathrm{c}}$ for fibers perpendicular to the heat flow is given by Verschoor et al. [6] as

$$
L_{c}=\frac{\pi}{4} \frac{D}{f}
$$

where $D$ is the mean diameter of the fiber distribution (according to the number distribution, discussed later), and $f$ is the fractional fiber volume in the board.

\subsection{Gas-Solid Conduction}

The thermal conductivity determined by the Fourier law considers the interaction between the solid and gas conduction in the fibrous material. Two main approaches have been used for calculating the combined gas and solid conductivities through fibers arranged randomly in a plane perpendicular to the heat flow. One method uses the thermal network and semi-empirical relations based on the model by Verschoor et al. [6],

$$
k=f^{m} k_{s}+k_{g}
$$

where $k_{s}$ is the conductivity of the fiber, and $m$ is an empirical coefficient that depends on the number of fibers and the orientation in the perpendicular plane [22]. The model has been evaluated for $m=2$ for loose fibrous insulation 
made of alumina [4], $m=1.469$ for multilayer insulation consisting of gold-coated reflective foils separated by alumina fibrous insulation [23], and $m=3$ for alumina-silica fibers [24-25].

Bhattacharyya [14] proposed two models based on Fricke's [26] method for electrical conductivity and considered variable orientation of the fibers. For fibers oriented perpendicularly to the direction of heat flow,

$$
k=\left[1-\frac{1-\frac{k_{g}}{k_{s}}}{1+\frac{2\left(k_{g} / k_{s}\right) f}{1+k_{g} / k_{s}}}\right] k_{s} .
$$

For fibers oriented totally randomly in three dimensions,

$$
k=\left[1-\frac{1-\frac{k_{g}}{k_{s}}}{1+\frac{f\left(1+5\left(k_{g} / k_{s}\right)\right)}{3\left(1+k_{g} / k_{s}\right)}}\right] k_{s} .
$$

The Bhattacharyya model for perpendicularly oriented fibers was used in this study due to the availability of data.

\section{Radiative Properties}

In this paper, we investigated three cases involving the radiative properties of the fibers. In the first case, the fibers are assumed to be highly reflective. For the other two cases, Mie theory, assuming that each fiber is an infinite cylinder, was applied in two different ways: the rigorous formulation of Lee [8-11] and the model of Tong and Tien [7].

\subsection{Large Specularly Reflective Cylinders (LSR)}

A simple model for the absorption constant considering cylindrical randomly oriented fibers was proposed by Farnworth [18]. The model can be deduced supposing a large, opaque, and specularly reflecting particle. The absorption efficiency can be expressed as [16]:

$$
Q_{\mathrm{abs}}=\alpha_{\mathrm{f}}
$$

where $Q_{\text {abs }}$ is the absorption efficiency, and $\alpha_{\mathrm{f}}$ is the absorptivity of the fiber. The absorption coefficient for a cloud of specularly reflective large spheres is [17]:

$$
\kappa_{\lambda}=\varepsilon_{f} \pi D L_{f} N
$$

where $\kappa$ is the absorption coefficient, and $\varepsilon_{\mathrm{f}}$ and $L_{\mathrm{f}}$ are the emissivity and the length of the fiber, respectively. The emissivity was determined using Kirchhoff's law. The number of particles per volume, $N$, can be calculated from the fractional fiber volume and the fiber diameter though Eq. (22). For this study, the fiber diameter distribution of the insulating material was determined (described later), and the mean diameter was used.

$$
N=\frac{4 f}{\pi D^{2} L_{f}} .
$$


Finally,

$$
\kappa_{\lambda}=\frac{4 \varepsilon_{f \lambda} f}{D}
$$

Using an analogous consideration for the scattering coefficient $\sigma_{\mathrm{s} \lambda}$

$$
\sigma_{s \lambda}=\frac{4\left(1-\varepsilon_{f \lambda}\right) f}{D}
$$

The extinction coefficient is given by

$$
\beta_{\lambda}=\sigma_{s \lambda}+\kappa_{\lambda}
$$

\subsection{Mie Theory}

The scattering by a single infinite cylinder through solution of the Maxwell equations was presented by Kerker [27] and summarized by Lee et al. [28] as:

$$
\begin{gathered}
C_{e \lambda}=\frac{4}{k_{0}} \operatorname{Re}\left\{b_{0 I}+a_{0 I I}+2 \sum_{n=1}^{\infty}\left(b_{n I}+a_{n I I}\right)\right\}, \\
C_{s \lambda}=\frac{4}{k_{0}} \operatorname{Re}\left\{\left|b_{0 I}\right|^{2}+\left|a_{0 I I}\right|^{2}+2 \sum_{n=1}^{\infty}\left(\left|b_{n I}\right|^{2}+\left|b_{n I I}\right|^{2}+\left|a_{n I}\right|^{2}+\left|a_{n I I}\right|^{2}\right)\right\},
\end{gathered}
$$

where $C_{\mathrm{e} \lambda}$ is extinction cross section, $C_{\mathrm{s} \lambda}$ is the scattering cross section, $k_{0}$ is the wavenumber in the outer medium, and $a$ and $b$ are coefficients that are functions of the complex refractive index of the fiber, the incident angle, the radius of the fiber, and the wavelength. The scattering intensity is given by

$$
i_{\lambda}(\theta, \varphi)=\left|T_{11}\right|^{2}+\left|T_{12}\right|^{2}+\left|T_{21}\right|^{2}+\left|T_{22}\right|^{2}
$$

where $T_{i j}$ are the elements of the $T$ matrix, defined as:

$$
\begin{gathered}
T_{11}=b_{0 I}+2 \sum_{n=1}^{\infty} b_{n I} \cos (n \theta), \\
T_{12}=2 \sum_{n=1}^{\infty} a_{n I} \sin (n \theta), \\
T_{21}=2 \sum_{n=1}^{\infty} b_{n I} \sin (n \theta), \\
T_{22}=a_{0 I I}+2 \sum_{n=1}^{\infty} a_{n I I} \cos (n \theta) .
\end{gathered}
$$




\subsubsection{Tong and Tien Model}

A fibrous insulator can be represented as a set of infinite cylinders randomly oriented in a plane. Tong and Tien modeled the fibers as homogeneous and infinitely long cylinders [7]. The extinction and scattering coefficients were calculated from:

$$
\begin{gathered}
\beta_{\lambda}=\frac{2 f}{\pi^{2} A} \int_{0}^{\pi / 2 \infty} \int_{0}^{\infty} C_{e \lambda} N_{f}(r) d r d \varphi, \\
\sigma_{s \lambda}=\frac{2 f}{\pi^{2} A} \int_{0}^{\pi / 2 \infty} \int_{0}^{2 \infty} C_{s \lambda} N_{f}(r) d r d \varphi, \\
A=\int_{0}^{\infty} r^{2} N_{f}(r) d r,
\end{gathered}
$$

where $r$ is the radius, $\varphi$ is the incident angle, and $N_{\mathrm{f}}$ is the distribution of the fiber size in the fibrous insulator.

\subsubsection{Lee Model}

Lee developed a rigorous formulation of the properties of fibrous media [8-11], including the fiber size, orientation in a plane, and two-dimensional (2D) characteristic of the radiation scattered by cylinders. The latter property is due to the constraint that radiation scattered by cylindrical fibers propagates along a conic surface [9].

$$
\begin{aligned}
& \beta_{\lambda}=\frac{f}{\pi} \sum_{j=1}^{N} \frac{x_{j}}{r_{j}^{2}} \int_{0}^{\pi / 2} C_{e \lambda} \cos \varphi d \varphi, \\
& \sigma_{s \lambda}=\frac{f}{\pi} \sum_{j=1}^{N} \frac{x_{j}}{r_{j}^{2}} \int_{0}^{\pi / 2} C_{s \lambda} \cos \varphi d \varphi,
\end{aligned}
$$

where $x_{j}$ is the fraction of fibers of radius $r_{j}$. Later, Lee proposed a modified extinction coefficient [13] calculated as

$$
\begin{gathered}
\bar{\beta}_{\lambda}=\beta_{\lambda}\left(1-G_{\lambda}\right), \\
G_{\lambda}=\frac{1}{\beta_{\lambda}} \int_{0}^{1} \int_{-1}^{1} \sigma_{s \lambda} p_{\lambda}\left(\mu, \mu^{\prime}\right) d \mu^{\prime} d \mu, \\
\sigma_{s \lambda} p_{\lambda}(\eta)=\frac{4 f \lambda}{\pi^{3}} \sum_{j=1}^{N} \frac{x_{j}}{r_{j}^{2}} \int_{0}^{1} \frac{i_{\lambda}(\eta, \varphi)}{\sqrt{(1-\cos \eta)\left(1+\cos \eta-2 \sin ^{2} \varphi\right)}} d(\sin \varphi), \\
\sigma_{s \lambda} p_{\lambda}\left(\mu, \mu^{\prime}\right)=\frac{1}{2 \pi} \int_{0}^{2 \pi} \sigma_{s \lambda} p_{\lambda}(\eta) d \omega,
\end{gathered}
$$

where $G_{\lambda}$ is the asymmetry scattering factor, $p$ is the phase scattering function, and $\eta$ is the scattering angle between the incident $(\mu, \omega)$ and scattered $\left(\mu^{\prime}, \omega^{\prime}\right)$ directions. 
The properties are deduced for a global coordinate system that is related to the fiber-centered coordinate system through [11]:

$$
\cos \eta=\mu^{\prime} \mu+\left[\left(1-\mu^{2}\right)\left(1-\mu^{\prime 2}\right)\right]^{1 / 2} \cos \left(\omega-\omega^{\prime}\right)
$$

In the Lee and Tong and Tien models, the extinction and scattering coefficients are functions of the wavelength. To account for the entire spectrum, the extinction coefficient is integrated over all wavelengths. For an optically thick medium, this leads to Eq. (43), commonly known as the Rosseland mean [17]:

$$
\frac{1}{\beta_{R}}=\int_{0}^{\infty}\left(\frac{1}{\beta_{\lambda}}\right)\left(\frac{\pi}{2} \frac{C_{1} C_{2}}{\lambda^{6}} \frac{\sigma^{1 / 4}}{E_{b}^{5 / 4}} \frac{\exp \left[\left(\frac{C_{2}}{\lambda}\right)\left(\frac{\sigma}{E_{b}}\right)^{1 / 4}\right]}{\left.\left[\exp \left[\left(\frac{C_{2}}{\lambda}\right)\left(\frac{\sigma}{E_{b}}\right)^{1 / 4}\right]-1\right]^{2}\right]} d \lambda\right.
$$

\section{Standard Reference Material 1450c}

NIST Standard Reference Material (SRM) 1450c is a high-density fibrous glass board utilized as a reference material for thermal resistance measurements. The thermal performance of several randomly picked samples has been characterized utilizing the NIST $1016 \mathrm{~mm}$ guarded-hot-plate apparatus [29]. The apparent thermal conductivity of SRM 1450c can be computed from the certification equation [30]:

$$
k_{\text {app }}=-7.2661 \times 10^{-3}+5.6252 \times 10^{-5} \rho+1.0741 \times 10^{-4} T \text {. }
$$

The certified values are valid from $150 \mathrm{~kg} \cdot \mathrm{m}^{-3}$ to $165 \mathrm{~kg} \cdot \mathrm{m}^{-3}$ and from $280 \mathrm{~K}$ to $340 \mathrm{~K}$. The nominal dimensions of each board are $25 \mathrm{~mm}$ in thickness by $610 \mathrm{~mm}$ by $610 \mathrm{~mm}$ [29]. The boards were manufactured by molding, under heat and pressure, layers of glass-fiber pelts treated with uncured binder. The fibers, which are an alkali-alkaline alumino-borosilicate glass with phenyl formaldehyde binder [29], are oriented randomly in layers parallel to the board faces and perpendicular to the direction of heat flow. Figure 2 is a scanning electron micrograph (SEM) of the material [29], which shows the orientation of the fibers in the plane of the image and evidence of small globules of binder among the fibers. It should be noted that Figure 2 shows the cross section of the specimen, so the fibers seem preferentially parallel oriented. However, within a layer, the fibers should be randomly oriented. Although visually informative, this image cannot provide the necessary quantitative data on the fiber diameter distribution. 


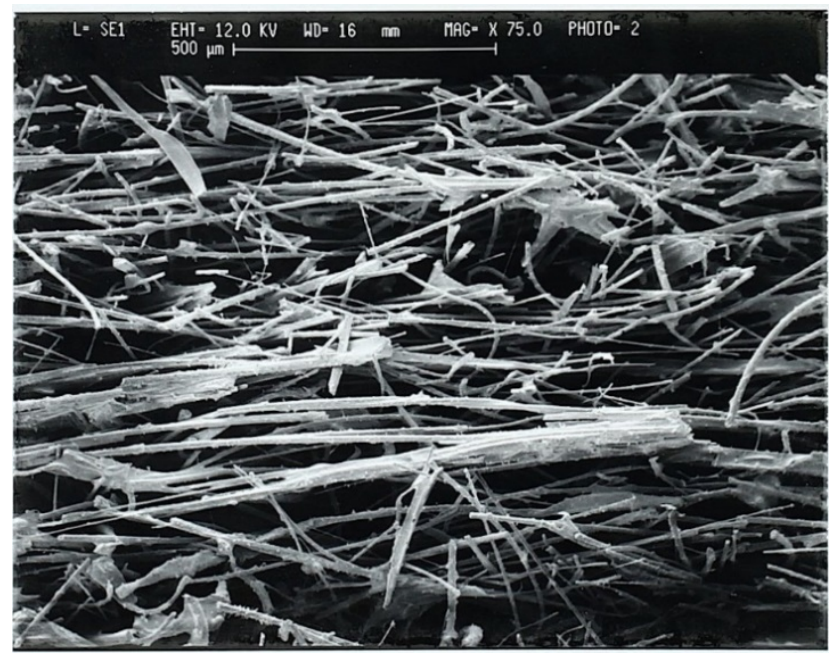

Fig. 2. SEM micrograph of part of SRM 1450c showing the shape of the fibers [29].

An X-ray computed tomography (CT) scanner was used to measure the fiber diameter distribution and compute the average fiber diameter, which is a parameter of interest in some of the thermal conduction theories used in this paper. A small piece (several millimeters in size) was scanned on a Skyscan $1172^{1} \mathrm{X}$-ray CT scanner. The sample was supported so that the vertical direction in the scanner was in the horizontal plane of the insulation. In this way, cross-sectional reconstructed images would intersect the fibers in many different directions. The first scan was taken in a mode such that the voxel size was $1.88 \mu \mathrm{m}$, and the reconstructed images were 2000 pixels $\times 2000$ pixels in size. There were 980 slices, so that the total size of the scanned section of the sample was approximately $3.6 \mathrm{~mm} \times$ $3.6 \mathrm{~mm} \times 1.76 \mathrm{~mm}$. A second scanning run, on the same sample in the same location, was made using a voxel size of $0.94 \mu \mathrm{m}$, and the 1959 reconstructed images were 4000 pixels $\times 4000$ pixels in size. This approach gave a similar physical sample size, so the same piece of insulation was examined but at approximate half the voxel size, so that smaller features were visible.

The goal was to measure the fiber diameter distribution. Each image was a cut through the random planar fiber orientation. For a cylindrical fiber, a cut through the fiber at a random angle, in general, produces an ellipse, with a circle of the same diameter being produced when the cut is perpendicular to the axis of the cylinder. For fibers of different diameters, it is not possible to distinguish between the ellipses produced from different diameter fibers and different angle cuts. Therefore, only the 2D "particles" in each image that were nearly circular were retained and stored, since these features were assumed to be perpendicular cuts through a fiber's axis. Each image was segmented to create a binary image, using a single threshold gray-scale value. For the 2000 pixel $\times 2000$ pixel images, $2 \mathrm{D}$ particles that were less than 16 pixels $\left(52 \mu \mathrm{m}^{2}\right)$ in area were discarded, since the shape of this size or smaller particle could not be determined accurately. This area corresponds to an equivalent circular diameter of about $8 \mu \mathrm{m}$, establishing the lower limit fiber diameter analyzed. Fibers that had a smaller diameter than this limit were not analyzed. Particles larger than 350 pixels in area $\left(1134 \mu \mathrm{m}^{2}\right)$ were also discarded, since these tended to be long, high-aspect-ratio particles and were assumed to be cross sections through fibers where the angle of cut was almost parallel to the fiber axis. Given this assumption, however, the fiber length could be estimated to be equal to the length of these larger objects, which was approximately 40 voxels to 250 voxels. A qualitative look at the images implied that the average fiber length was about 80 voxels or $150 \mu \mathrm{m}$. An ellipse was then fit to the remaining particles, and aspect ratios greater than 1.1 were discarded. The remaining particles were counted and measured, and their equivalent circular diameter was recorded. Figure 3 illustrates the image analysis procedure for a single 2000 pixel $\times 2000$ pixel cross-sectional slice.

${ }^{1}$ Certain commercial entities, equipment, or materials may be identified in this document in order to describe an experimental procedure or concept adequately. Such identification does not imply recommendation or endorsement by the National Institute of Standards and Technology (NIST), nor does it imply that the entities, materials, or equipment are necessarily the best available for the purpose. 

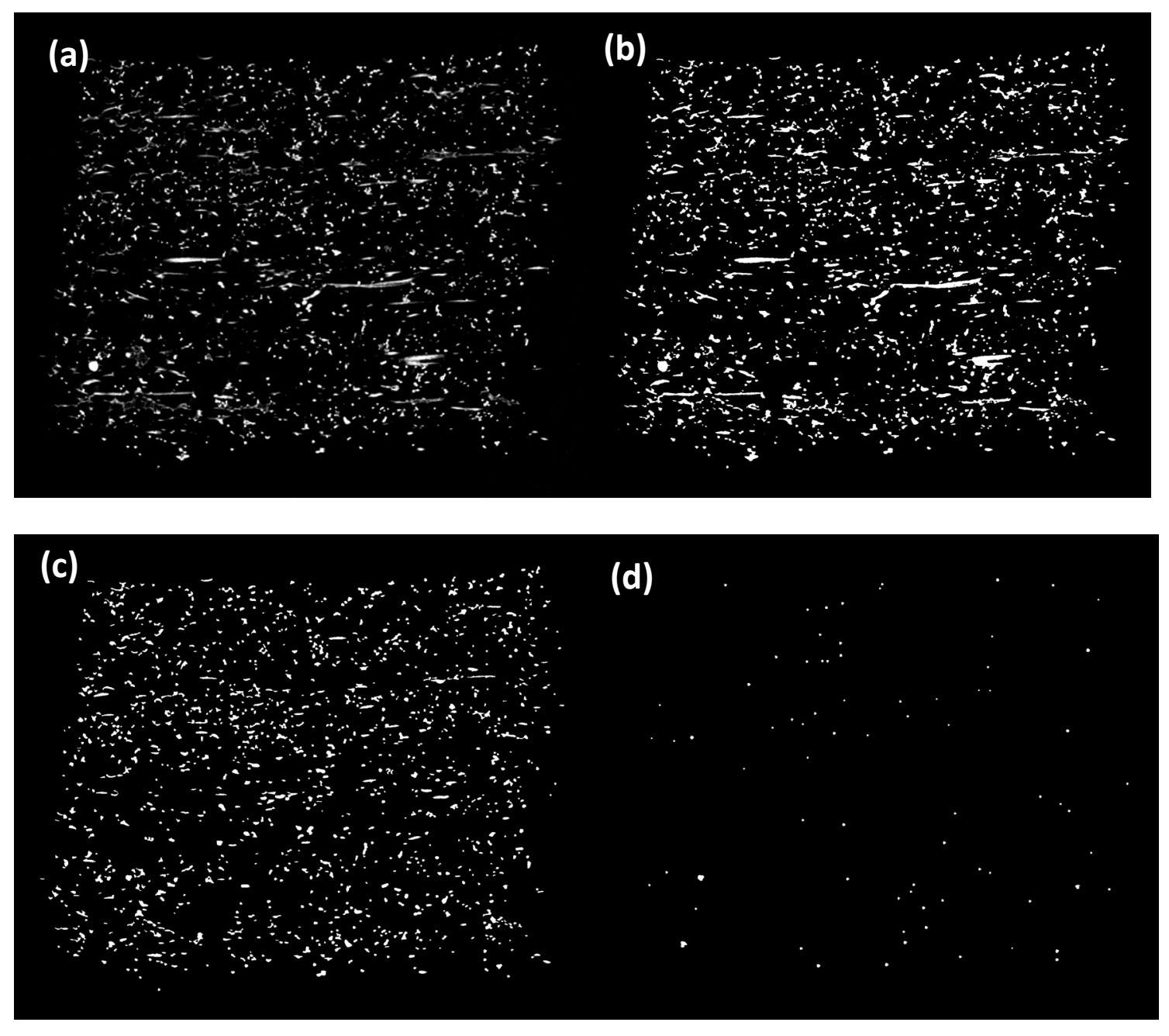

Fig. 3. (a) Single gray-scale 2000 pixel $\times 2000$ pixel reconstructed slice, (b) segmented image, (c) "particles" with areas $>350$ pixels and areas $<$ 16 pixels removed, and (d) "particles" with aspect ratios $>1.1$ removed.

Figure 4 shows the differential distribution histogram that was found for these objects, in terms of the fiber number. The average fiber diameter was found to be $14.0 \mu \mathrm{m} \pm 3.9 \mu \mathrm{m}$, where the uncertainty is based on one standard deviation. This uncertainty is not an experimental uncertainty, but simply a reflection of the distribution of fiber diameter. The actual experimental uncertainty comes from segmentation and is equal to about one pixel length, which is about $2 \mu \mathrm{m}$ for the 2000 pixel $\times 2000$ pixel images. Since every slice was analyzed, each fiber that was aligned with the vertical dimension of the sample had its cross section averaged along its length, which on average was approximately 80 slices. So, the 81492 data points obtained from the 2000 pixel $\times 2000$ pixel images included many duplicates from the same fiber. If we use the above rough estimate of average fiber length, then there were, on average, about 80 slices per fiber, or about $81492 / 80 \approx 1000$ distinct fibers that were actually measured. The computed average diameter includes the averaging of each fiber along its cross section, so that any variability in each fiber's diameter is included in the average fiber diameter recorded here.

The graph includes similar results taken from the set of 4000 pixel $\times 4000$ pixel images. The same lower area filter bound, 16 pixels in area $\left(14 \mu \mathrm{m}^{2}\right)$, with an equivalent circular diameter of about $4 \mu \mathrm{m}$, was used, but the upper area bound was extended to 1400 pixels in area to obtain about the same physical size for the upper fiber range as 
before. Because smaller fibers could be resolved at this smaller pixel size, the average fiber diameter was expected to decrease. If we take the total number of data points, 227736 , divided by the average pixel length of the fibers (about twice the previous estimate, since half the pixel size), this implies about 1400 unique fiber diameters were measured, which is more than the 2000 pixel $\times 2000$ pixel result, because smaller fibers could now be measured. Since the 400 more fibers measured must have all been in the $4 \mu \mathrm{m}$ to $8 \mu \mathrm{m}$ diameter range, these extra fibers lowered the average fiber diameter significantly. The new average obtained was $12.4 \mu \mathrm{m} \pm 3.7 \mu \mathrm{m}$. The experimental uncertainty was again about one pixel, or $1 \mu \mathrm{m}$.

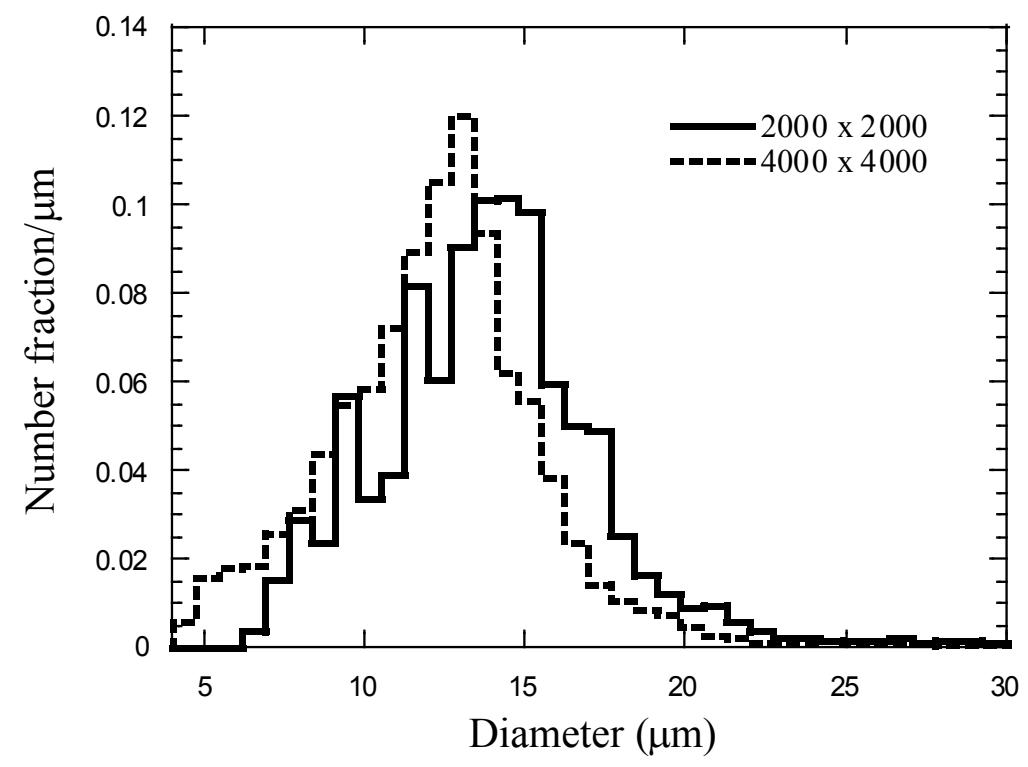

Fig. 4. Fiber size distribution of SRM 1450c, based on number weighting.

The diameter distribution for the 4000 pixel $\times 4000$ pixel results depicted in Fig. 4 was used to compute the radiative properties in Eqs. (33) through (35) and Eqs. (36) through (41). The average diameter was taken as $12.4 \mu \mathrm{m} \pm 3.7 \mu \mathrm{m}$ and the average aspect ratio of the fibers was estimated to be 12 . Understandably, the theories reviewed in this paper that assume the fibers are infinite cylinders will be subject to error (albeit small) due to the fact that the fibers actually have finite lengths.

\section{Results and Discussion}

Each model reviewed in Table 2 was evaluated using the RTE approximations summarized in Table 3 . The Lee model, however, was evaluated only with the diffusion approximation due to the asymmetry factor defined for this radiative transfer equation. The coupled differential equations were solved using the finite difference method. As emphasized by Lee [9], special care must be taken in the integration of Eq. (40) due the limits of integration. The scattering region is defined by the line $\cos \eta=1$ and the parabola $\cos \eta=2 \cos ^{2}(\pi-2 \varphi)-1$; however, the expression inside the square root in Eq. (40) is not continuous for $\varphi$ between $\pi / 3$ and $\pi / 2$ and for $\omega$ between $\pi$ and $2 \pi$. To avoid the discontinuity, Eq. (41) was integrated between 0 and $\pi$ assuming symmetry in the azimuthal angle. 


\section{Journal of Research of the National Institute of Standards and Technology}

Table 2. Summary of models.

\begin{tabular}{lccc}
\hline \multicolumn{1}{c}{ Property } & $\begin{array}{c}\text { Large specular } \\
\text { reflective cylinders (LSR) }\end{array}$ & Tong and Tien [7] & Lee [8-11] \\
\hline Gas phase conductivity & Eqs. (14) through (16) & Eqs. (14) through (16) & Eqs. (14) through (16) \\
Solid phase conductivity & Eq. (18) & Eq. (18) & Eq. (18) \\
Extinction coefficient & Eq. (25) & Eqs. (33) through (35) & Eqs. (36) through \\
\hline
\end{tabular}

Table 3. Summary of radiative transfer equations.

\begin{tabular}{ccc}
\hline Diffusion & Schuster and Schwarzschild (SS) & Milne-Eddington (ME) \\
\hline Eq. (4) & Eqs. (5) through (10) & Eqs. (11) through (13) \\
\hline
\end{tabular}

To evaluate the validity of the models in Table 2 , predicted values were compared to the certified values of SRM 1450c determined from Eq. (44). The required thermophysical properties in the models are presented in Table 4. The complex refractive index was taken between wavelengths of $0.32 \mu \mathrm{m}$ and $206.6 \mu \mathrm{m}$ from Hsieh [30]. The relative errors were computed for different input values of temperature and bulk density. Comparisons among the evaluated models and certified values for SRM 1450c from Eq. (44) are presented graphically in Figs. 5 through 10.

Table 4. Thermophysical properties.

\begin{tabular}{|c|c|c|c|c|c|}
\hline $\begin{array}{l}\text { Fiber density } \\
\left(\mathrm{kg} \cdot \mathrm{m}^{-3}\right)\end{array}$ & $\begin{array}{l}\text { Bulk density } \\
\left(\mathrm{kg} \cdot \mathrm{m}^{-3}\right)\end{array}$ & $\begin{array}{l}\text { Fiber thermal } \\
\text { conductivity } \\
\left(\mathrm{W} \cdot \mathrm{m}^{-1} \cdot \mathrm{K}^{-1}\right)\end{array}$ & $\begin{array}{l}\text { Air thermal } \\
\text { conductivity } \\
\left(\mathrm{W} \cdot \mathrm{m}^{-1} \cdot \mathrm{K}^{-1}\right)\end{array}$ & $\begin{array}{l}\text { Fractional } \\
\text { fiber volume }\end{array}$ & $\begin{array}{c}\text { Characteristic length } \\
(\mu \mathrm{m})\end{array}$ \\
\hline \multirow{7}{*}{2230} & 150.0 & \multirow{7}{*}{1.14} & \multirow{7}{*}{$8 \mathrm{E}-5 T+0.0031$} & 0.067 & 144.44 \\
\hline & 152.5 & & & 0.068 & 142.07 \\
\hline & 155.0 & & & 0.070 & 139.78 \\
\hline & 157.5 & & & 0.071 & 137.56 \\
\hline & 160.0 & & & 0.072 & 135.41 \\
\hline & 162.5 & & & 0.073 & 133.32 \\
\hline & 165.0 & & & 0.074 & 131.30 \\
\hline
\end{tabular}

Figures 5, 6, and 7 plot effective thermal conductivity of a high-density fibrous insulation $\left(155.5 \mathrm{~kg} \cdot \mathrm{m}^{-3}\right)$ as a function of temperature for the three cases involving radiative transfer equations (Table 3): diffusion, Schuster and Schwarzschild, and Milne-Eddington approximations, respectively. Certified values for SRM 1450c, determined from Eq. (44), are plotted as solid data points in increments of $5 \mathrm{~K}$ from $280 \mathrm{~K}$ to $340 \mathrm{~K}$. The vertical error bars represent an expanded uncertainty of $\pm 1.6 \%$ (coverage factor equal to 2 ). The gas-solid conduction contribution from Eq. (18) was also plotted to evaluate the influence of thermal radiation.

As is evident in each plot, predicted values for the models over the limited temperature range of $280 \mathrm{~K}$ to $340 \mathrm{~K}$ are linear or nearly linear. Figure 5 shows that the three models that use the diffusion approximation all underpredict the certified values, although the Lee model is quite close. The relative errors for the Lee model improve with temperature, from approximately $4 \%$ difference at $280 \mathrm{~K}$ to approximately $2 \%$ difference from the certified values at $340 \mathrm{~K}$. The LSR model diverges from the certified values with increasing temperature, increasing to approximately $8.5 \%$ at $340 \mathrm{~K}$. Figure 6 shows that the two models that used the Schuster and Schwarzschild approximation overpredict the certified values. The relative errors increase from $12 \%$ at $280 \mathrm{~K}$ to $20 \%$ at $340 \mathrm{~K}$. Figure 7 shows that the two models that used the Milne-Eddington approximation underpredict the certified values. The slopes are nearly the same, and the relative error is offset by approximately $4 \%$ over the temperature interval. 


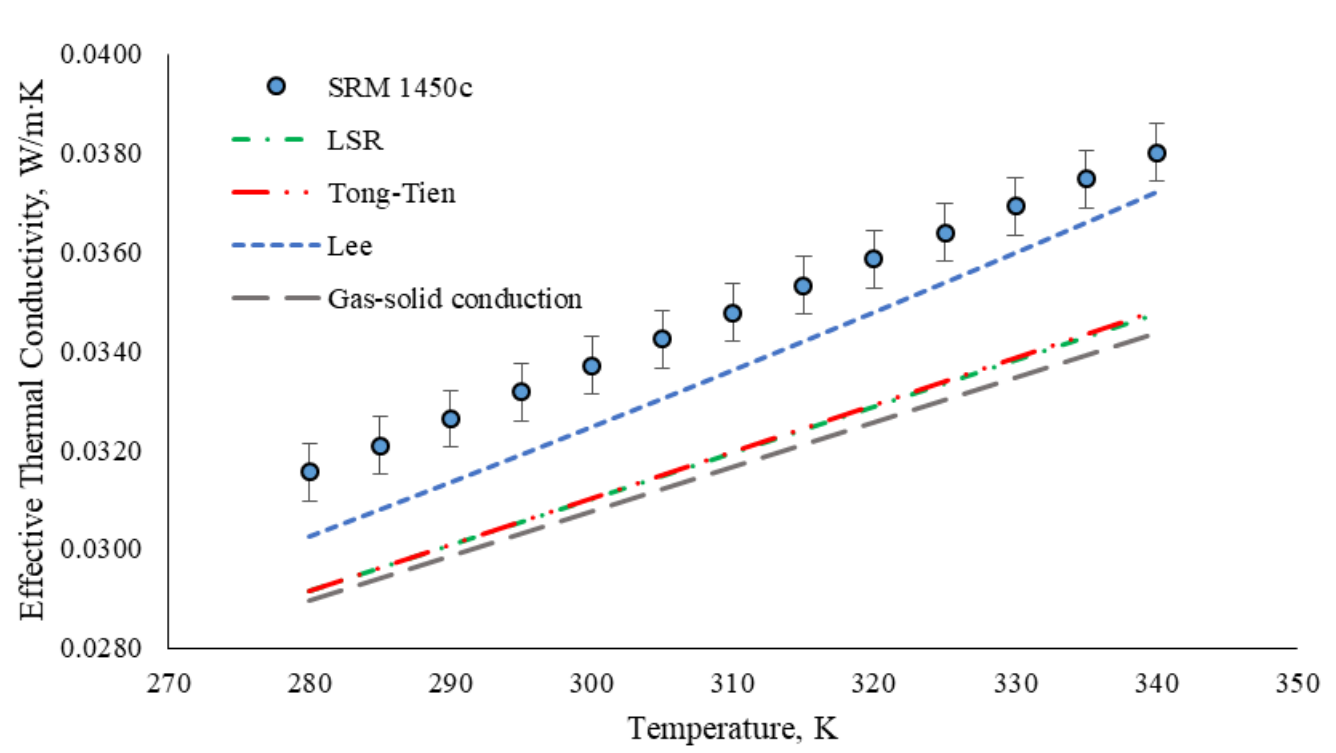

Fig. 5. Comparison among SRM 1450c certified values and predicted values using the diffusion approximation at a density of $155.5 \mathrm{~kg} \cdot \mathrm{m}^{-3}$.

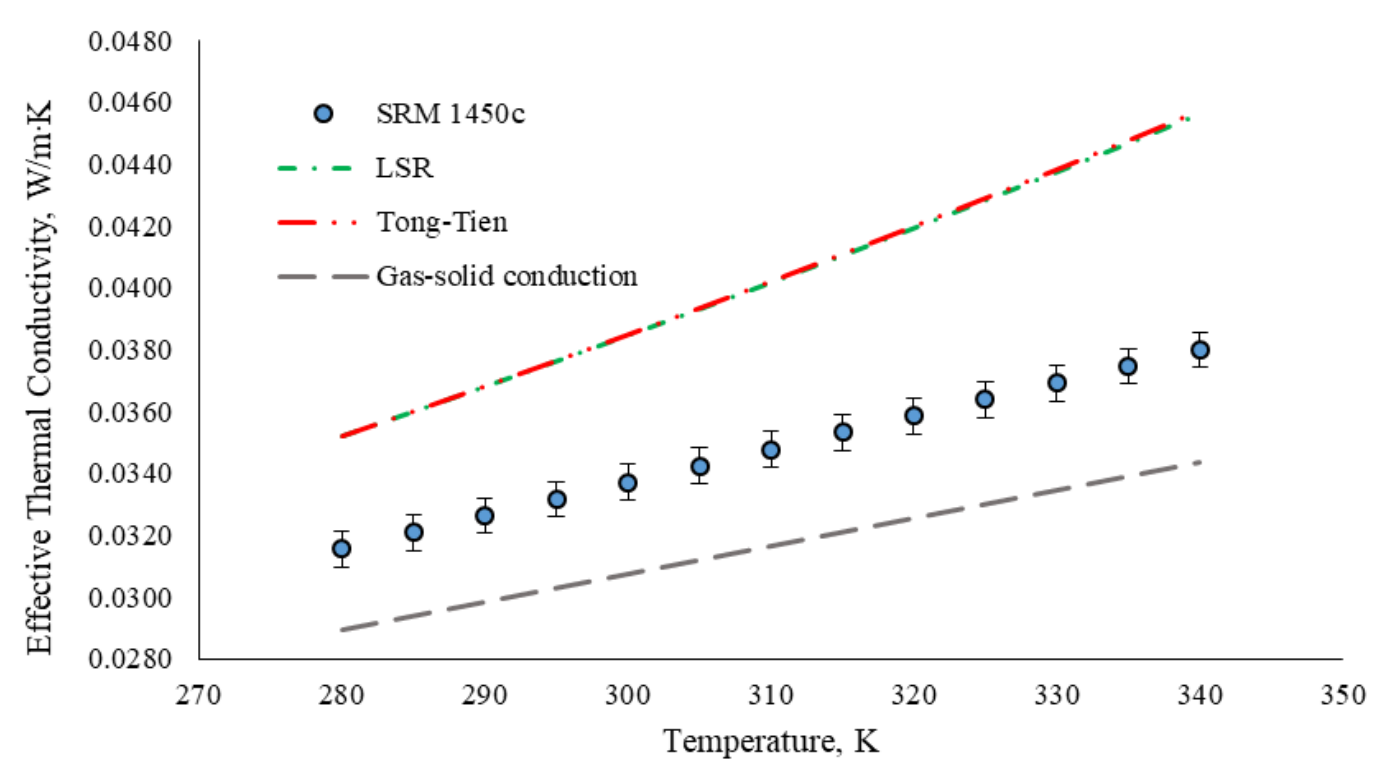

Fig. 6. Comparison among SRM 1450c and predicted values using the Schuster and Schwarzschild approximation at $155.5 \mathrm{~kg} \cdot \mathrm{m}^{-3}$. 


\section{Journal of Research of the National Institute of Standards and Technology}

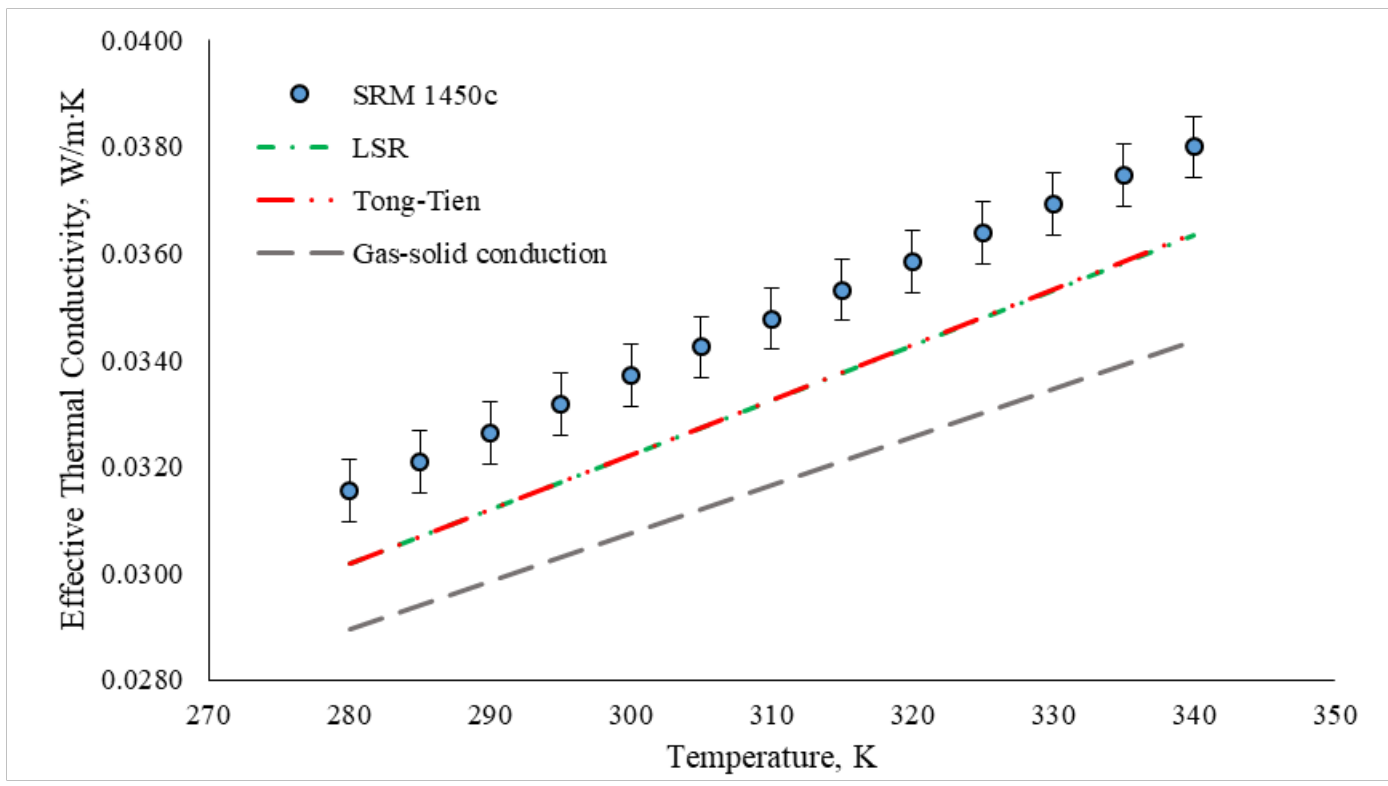

Fig. 7. Comparison among SRM $1450 \mathrm{c}$ and predicted values using the Milne-Eddington approximation at $155.5 \mathrm{~kg} \cdot \mathrm{m}^{-3}$.

To compare the dependence of the thermal conductivity with bulk density, the models were evaluated at constant temperature over a bulk density range from $150 \mathrm{~kg} \cdot \mathrm{m}^{-3}$ to $165 \mathrm{~kg} \cdot \mathrm{m}^{-3}$. Figures 8,9 , and 10 plot effective thermal conductivity at $295 \mathrm{~K}$ for a high-density fibrous insulation as a function of bulk density for the three cases involving radiative transfer equations: diffusion, Schuster and Schwarzschild, and Milne-Eddington approximations, respectively. Certified values for SRM 1450c are plotted as solid data points in increments of $2.5 \mathrm{~kg} \cdot \mathrm{m}^{-3}$ from 150 $\mathrm{kg} \cdot \mathrm{m}^{-3}$ to $165 \mathrm{~kg} \cdot \mathrm{m}^{-3}$. Again, the vertical error bars represent an expanded uncertainty of $\pm 1.6 \%$ (coverage factor equal to 2).

As was observed in the previous plots, over the limited density range of $150 \mathrm{~kg} \cdot \mathrm{m}^{-3}$ to $165 \mathrm{~kg} \cdot \mathrm{m}^{-3}$, predicted values for the three cases are linear, or nearly linear, and show a slight positive correlation with density. In general, the results of Figs. 8 through 10 depict similar trends in model prediction relative to the certified values as was observed in Figs. 5 through 7. The results of Fig. 8 show that the Lee model is in close agreement with the certified values. Interestingly, the Lee model agrees better at lower densities, on the order of $3 \%$ at $150 \mathrm{~kg} \cdot \mathrm{m}^{-3}$, and increases to $5 \%$ at $165 \mathrm{~kg} \cdot \mathrm{m}^{-3}$. The relative errors of the other two models range from $7 \%$ to $9 \%$ over the density range. The results of Fig. 9 show that the two models overpredict the effective thermal conductivity, from $14 \%$ at $150 \mathrm{~kg} \cdot \mathrm{m}^{-3}$ to $12 \%$ at $165 \mathrm{~kg} \cdot \mathrm{m}^{-3}$. The results of Fig. 10 show that the two models underpredict the effective thermal conductivity, from $4 \%$ at $150 \mathrm{~kg} \cdot \mathrm{m}^{-3}$ to $5 \%$ at $165 \mathrm{~kg} \cdot \mathrm{m}^{-3}$.

For the temperature interval of $280 \mathrm{~K}$ to $340 \mathrm{~K}$, the model by Lee (Fig. 5), using the modified extinction coefficient and the diffusion approximation, best represents the certified values of SRM 1450c. This result is somewhat unanticipated, because the radiative properties models assume that the fibers are infinitely long, even though the fibers have been measured to have an aspect ratio of about 10 (as described above). The success of the Lee model relies in the computation of the two-dimensional scattering, which reduces the extinction coefficient by the factor $\left(1-G_{\lambda}\right)$. The diffusion approximation, when used with either the LSR model or the Tong-Tien model, shows similar relative differences. However, the variation in the relative difference over the temperature range is lower than in the Lee model. 


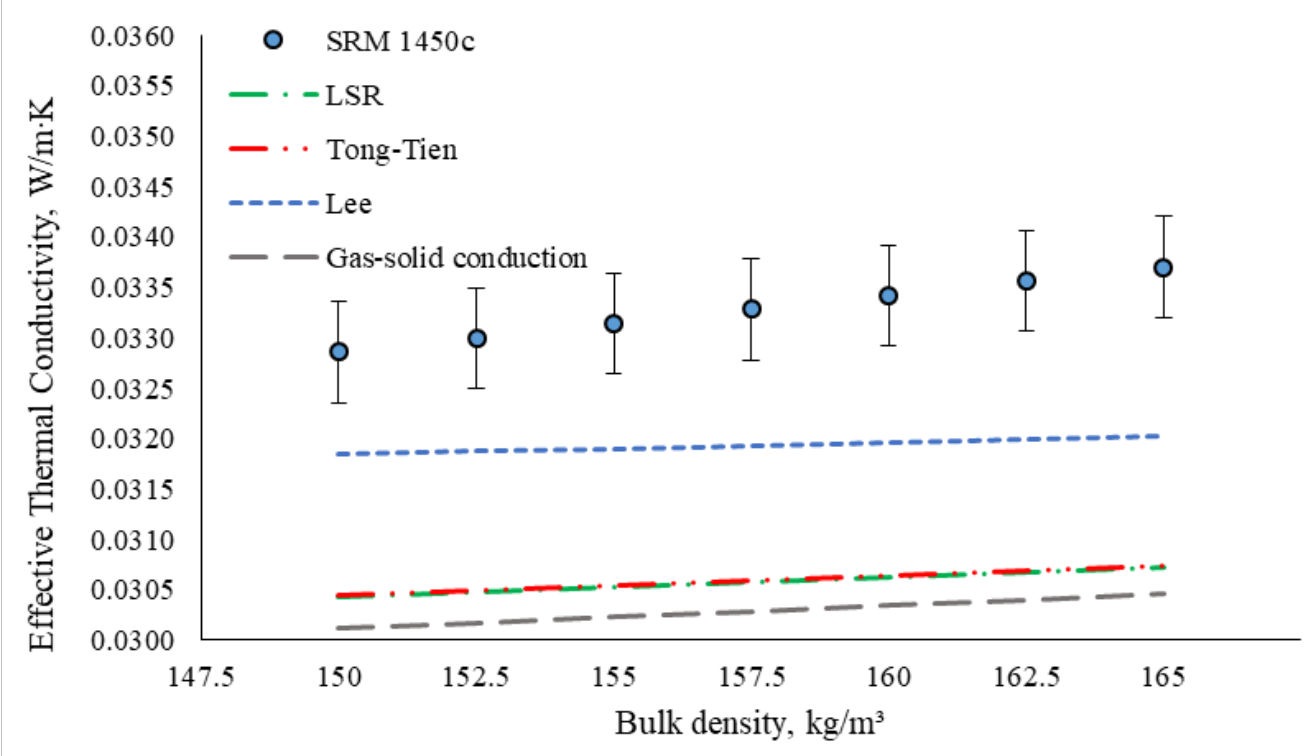

Fig. 8. Comparison among SRM 1450c and predicted values using the diffusion approximation at $295 \mathrm{~K}$.

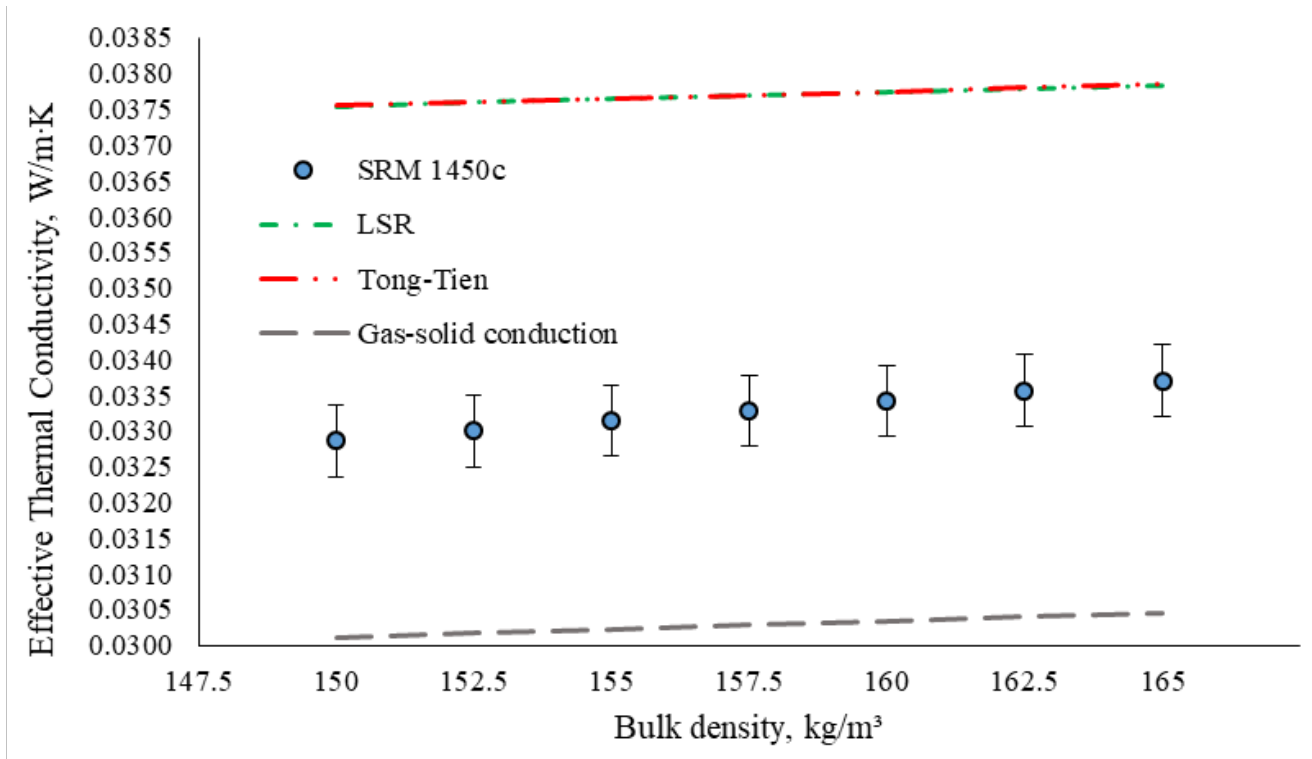

Fig. 9. Comparison among SRM 1450c and predicted values using the Schuster and Schwarzschild approximation at $295 \mathrm{~K}$. 


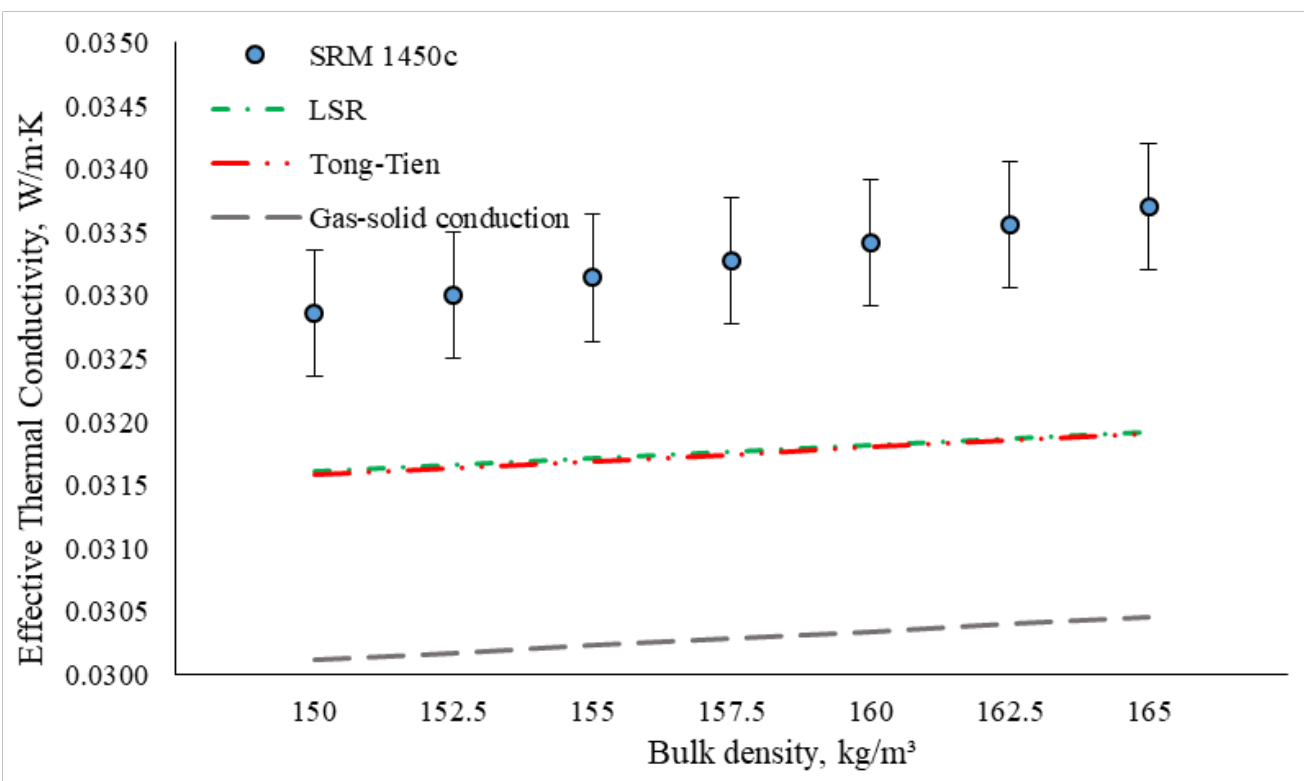

Fig. 10. Comparison among SRM 1450c and predicted values using the Milne-Eddington approximation at $295 \mathrm{~K}$.

The Schuster and Schwarzschild approximation (Fig. 6) shows similar results when used with either the LSR model or Tong-Tien model. The divergence of predicted values from the certified values of SRM 1450c at the higher temperatures is probably related to the supposition of no scattering. In fibrous glass insulation, scattering is expected to dominate absorption, as shown by Larkin and Churchill [31]. The Milne-Eddington approximation (Fig. 7) shows similar results when used with either the LSR model or Tong-Tien model. In this case, the relative differences from the certified values are comparable to the errors obtained with the Lee model (Fig. 5) at temperatures between $280 \mathrm{~K}$ and $300 \mathrm{~K}$, although the variation in relative error is less over the entire temperature interval evaluated. The average slope for the Milne-Eddington approximation (Fig. 7) was $1.0198 \times 10^{-4} \mathrm{~W} /\left(\mathrm{m} \cdot \mathrm{K}^{2}\right)$, which is in good agreement with the certified slope of $1.0741 \times 10^{-4} \cdot \mathrm{W} /\left(\mathrm{m} \cdot \mathrm{K}^{2}\right)$ from Eq. (44). The results of the Milne-Eddington approximation can be associated with the inclusion of the absorption and scattering factors in the radiative transfer equation.

As observed in Figs. 8, 9, and 10, the effective thermal conductivity is a weak (linear) function of bulk density over the range of $150 \mathrm{~kg} \cdot \mathrm{m}^{-3}$ to $165 \mathrm{~kg} \cdot \mathrm{m}^{-3}$. The slope for the certified values of thermal conductivity is $5.6252 \times 10^{-5}\left(\mathrm{~W} \cdot \mathrm{m}^{2}\right) /(\mathrm{kg} \cdot \mathrm{K})$ from Eq. (44), and the slopes from Figs. 8, 9, and 10 are all approximately $2 \times 10^{-5}\left(\mathrm{~W} \cdot \mathrm{m}^{2}\right) /(\mathrm{kg} \cdot \mathrm{K})$.

It should be noted that all the models proposed for the estimation of the radiative properties assume a large aspect ratio; that is, the fibers are essentially infinitely long. Results from the X-ray CT scanner, however, determined an aspect ratio rounded to an order of magnitude of 10 . However, in this study, the radiative terms were small in comparison with the conduction contribution. All the models evaluated (Table 3) showed coherent estimation of the radiative properties, especially with the diffusion approximation and the Milne-Eddington approximation.

In summary, the model proposed by Lee produces excellent agreement with the certified values of SRM 1450c, which can be attributed to the effect of dependent scattering and the effect of the fiber orientation in the material. The model by Tong and Tien neglects these effects and produced similar results with the LSR method, which is based on the approximation of large particles. Even so, the relative errors for the other models are less than $20 \%$ and, in some cases, less than $5 \%$. 


\section{Conclusions}

No fitting parameters, but only measured physical and structural properties of the specimen, were used to calculate the temperature-dependent thermal conductivity of NIST SRM 1450c, Fibrous Glass Board. All approaches, except for the nonscattering model, agreed with the experimental data to within $9 \%$ relative error, or less. The relative error for a given model, however, was either positive or negative in sign. In high-density fibrous insulators near ambient temperature, the main mechanism of heat transfer is conduction in the gas and solid fibers. The treatment of radiative heat transfer has only a minor impact on the calculated thermal conductivity.

X-ray CT was used to measure the fiber diameter distribution (number-based) and an approximate fiber aspect ratio of 12 . Since the radiative terms were small, using radiation theory for infinite cylinders instead of 10-to-1 aspect ratio cylinders did not introduce much error in the various models that calculated radiative terms.

The Lee model for optical properties with the diffusion approximation agreed well with the certified values of SRM 1450c (relative errors of $2 \%$ to $5 \%$ ) due to the rigorous formulation of the radiative process, which considered the fiber diameter distribution, random fiber orientation, and two-dimensional scattering. The LSR and Tong and Tien models offer comparable estimation for the optical properties. The relative difference found was between $7 \%$ and $9 \%$.

The diffusion approximation for radiative heat transfer in fibrous insulation closely agreed with the data near ambient temperature and pressure. The Schuster and Schwarzschild model overestimated the effective thermal conductivity by $12 \%$ to $20 \%$. This model assumed no radiation scattering, which is not expected to be the case in fibrous insulation materials such as SRM 1450c, where the fibers are closely packed. The Milne-Eddington approximation offers better estimation when the radiative properties are calculated from the LSR and Tong and Tien models.

\section{Appendix}

The calculation procedures for radiative properties in the Tong and Tien model and in the Lee model are documented in the flow charts in Figs. 11 and 12, respectively.

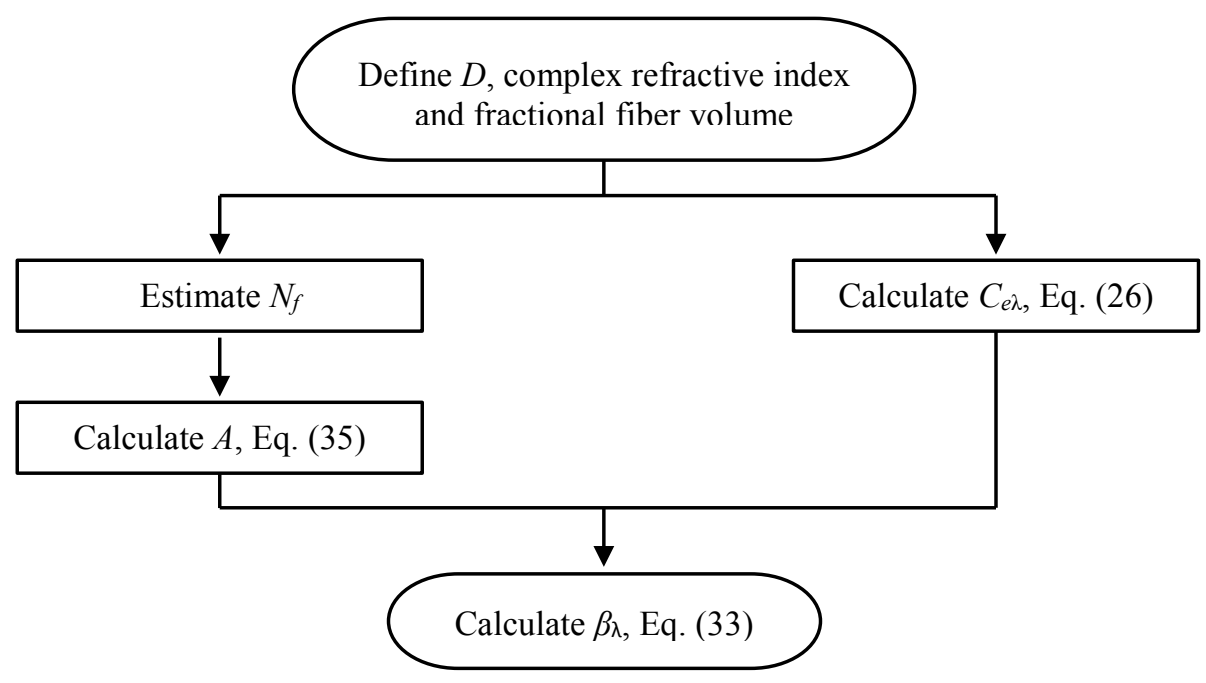

Fig. 11. Flow chart for the calculation of the extinction coefficient in the Tong and Tien model [7]. 


\section{Journal of Research of the National Institute of Standards and Technology}

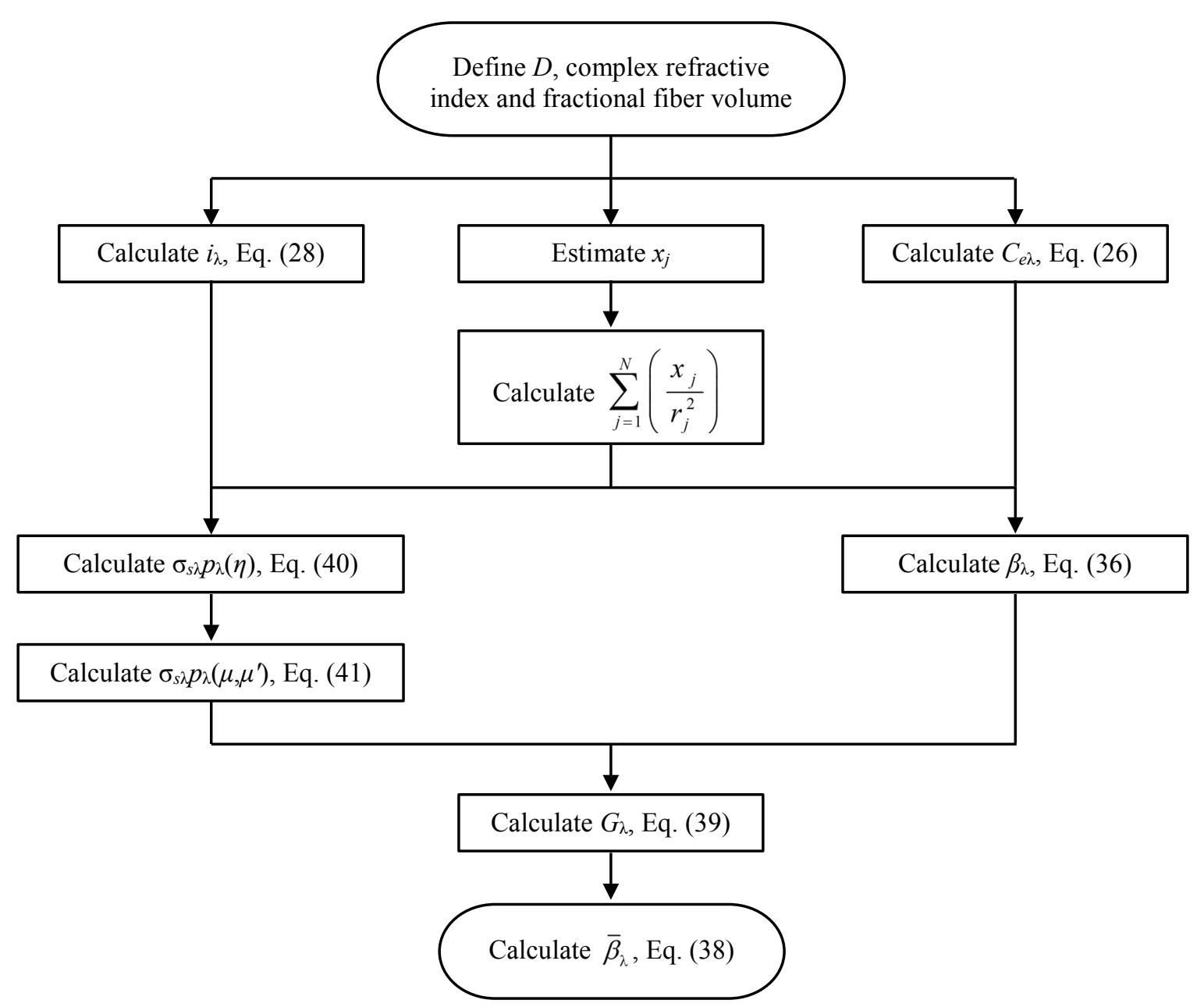

Fig. 12. Flow chart for the calculation of the extinction coefficient in the Lee model [8-11].

\section{References}

[1] Lee SC, Cunnington GR Jr (1998) Theoretical Models for radiative transfer in fibrous media. Annual Review of Heat Transfer 9(9):159-218. https://doi.org/10.1615/AnnualRevHeatTransfer.v9.50

[2] Stephenson DG (2009) A numerical procedure for calculating combined conduction and radiation heat flux through fibrous insulation. Journal of Building Physics 33(3):271-295. https://doi.org/10.1177/1744259109350658

[3] Zhang B-M, Zhao S-Y, He X-D (2008) Experimental and theoretical studies on high-temperature thermal properties of fibrous insulation. Journal of Quantitative Spectroscopy and Radiative Transfer 109(7):1309-1324. https://doi.org/10.1016/j.jqsrt.2007.10.008

[4] Daryabeigi K (2003) Heat transfer in high-temperature fibrous insulation. Journal of Thermophysics and Heat Transfer 17(1):10-20. https://doi.org/10.2514/2.6746

[5] Van Poolen LJ, Hust JG, Smith DR (1983) A model of apparent thermal conductivity for glass-fiber insulations. Thermal Conductivity, ed Hust JG (Plenum, New York, NY), pp. 777-788.

[6] Verschoor JD, Greebler P (1952) Heat transfer by gas conduction and radiation in fibrous insulation. Transactions of the American Society of Mechanical Engineers 74:961-968.

[7] Tong TW, Tien CL (1980) Analytical models for thermal radiation in fibrous insulations. Journal of Building Physics 4(1):27-44. https://doi.org/10.1177/109719638000400102

[8] Lee SC (1988) Radiation heat-transfer model for fibers oriented parallel to diffuse boundaries. Journal of Thermophysics and Heat Transfer 2(4):303-308. https://doi.org/10.2514/3.104

[9] Lee SC (1990) Scattering phase function for fibrous media. International Journal of Heat and Mass Transfer 33(10):2183-2190. https://doi.org/10.1016/0017-9310(90)90119-F

[10] Lee SC (1989) Effect of fiber orientation on thermal radiation in fibrous media. International Journal of Heat and Mass Transfer 32(2):311-319. https://doi.org/10.1016/0017-9310(89)90178-6

[11] Lee SC (1986) Radiative transfer through a fibrous medium: Allowance for fiber orientation. Journal of Quantitative Spectroscopy and Radiative Transfer 36(3):253-263. https://doi.org/10.1016/0022-4073(86)90073-7 


\section{Journal of Research of the National Institute of Standards and Technology}

[12] Raed K, Gross U (2008) Review on gas thermal conductivity in porous materials and Knudsen effect. Thermal

Conductivity 29/Thermal Expansion 17, Koenig JR, Ban H, eds (Destech Publications Inc., Lancaster, PA, USA), pp 356-373.

[13] Lee SC, Cunnington GR (1998) Heat transfer in fibrous insulations: Comparison of theory and experiment. Journal of Thermophysics and Heat Transfer 12(3):297-303. https://doi.org/10.2514/2.6356

[14] Bhattacharyya RK (1980) Heat transfer model for fibrous insulations. Thermal Insulation Performance. McElroy DL, Tye RP, eds (American Society for Testing and Materials, West Conshohocken, PA), ASTM STP 718, pp 272-286. https://doi.org/10.1520/STP29279S

[15] Stark C, Fricke J (1993) Improved heat-transfer models for fibrous insulations. International Journal of Heat and Mass Transfer 36(3):617-625. https://doi.org/10.1016/0017-9310(93)80037-U

[16] Modest MF (2013) Radiative Heat Transfer (Elsevier Science, San Diego, CA), 3rd Ed.

[17] Howell JR, Menguc MP, Siegel R (2010) Thermal Radiation Heat Transfer (CRC Press, Boca Raton, FL), 5th Ed.

[18] Farnworth B (1983) Mechanisms of heat flow through clothing insulation. Textile Research Journal 53(12):717-725. https://doi.org/10.1177/004051758305301201

[19] Du N, Fan J, Wu H (2008) Optimum porosity of fibrous porous materials for thermal insulation. Fibers and Polymers 9(1):27-33. https://doi.org/10.1007/s12221-008-0005-5

[20] Mavromatidis LE, Michel P, El Mankibi M, Santamouris M (2010) Study on transient heat transfer through multilayer thermal insulation: Numerical analysis and experimental investigation. Building Simulation 3(4):279-294. https://doi.org/10.1007/s12273-0100018-z

[21] Daryabeigi K (2001) Thermal analysis and design of multi-layer insulation for re-entry aerodynamic heating. 35th AIAA Thermophysics Conference, AIAA 2001-2834. https://doi.org/10.2514/6.2001-2834

[22] Williams SD, Curry DM (1993) Prediction of Rigid Silica Based Insulation Conductivity. (National Aeronautics and Space Administration, Washington, D.C.), NASA Technical Paper 3276.

[23] Daryabeigi K, Miller S, Cunnington G (2006) Heat transfer in high-temperature multilayer insulation. 5th European Workshop on Thermal Protection Systems and Hot Structures, $\mathrm{p} 631$.

[24] Daryabeigi K (1999) Analysis and testing of high temperature fibrous insulation for reusable launch vehicles. 37th Aerospace Sciences Meeting and Exhibit, p 1044. https://doi.org/10.2514/6.1999-1044

[25] Hager NE Jr, Steere RC (1967) Radiant heat transfer in fibrous thermal insulation. Journal of Applied Physics 38(12):4663-4668. https://doi.org/10.1063/1.1709200

[26] Fricke H (1924) A mathematical treatment of the electric conductivity and capacity of disperse systems I. The electric conductivity of a suspension of homogeneous spheroids. Physical Reivew 24(5):575-587. https://doi.org/10.1103/PhysRev.24.575

[27] Kerker M, Loebl EM (2000) The Scattering of Light and Other Electromagnetic Radiation (Elsevier Science, New York, NY). https://doi.org/10.1016/C2013-0-06195-6

[28] Lee SC, Cunnington GR (2000) Conduction and radiation heat transfer in high-porosity fiber thermal insulation. Journal of Thermophysics and Heat Transfer 14(2):121-136. https://doi.org/10.2514/2.6508

[29] Zarr RR (1997) Standard Reference Materials: Glass Fiberboard, SRM 1450c, for Thermal Resistance from 280 K to 340 K. (National Institute of Standards and Technology, Gaithersburg, MD), NIST Special Publication (SP) 260-130. https://doi.org/10.6028/NIST.SP.260-130

[30] Hsieh CK, Su KC (1979) Thermal radiative properties of glass from 0.32 to $206 \mu \mathrm{m}$. Solar Energy 22(1):37-43. https://doi.org/10.1016/0038-092X(79)90057-4

[31] Larkin BK, Churchill SW (1959) Heat transfer by radiation through porous insulations. AIChE Journal 5(4):467-474. https://doi.org/10.1002/aic.690050413

About the authors: Sergio A. Carvajal is a chemical engineer in the Temperature and Humidity Laboratory of the Instituto Nacional de Metrologia de Colombia, Bogotá D.C., Colombia.

Edward J. Garboczi is a NIST Fellow with the Applied Chemicals and Materials Division in the Material Measurement Laboratory.

Robert R. Zarr is a mechanical engineer in the Energy and Environment Division of the NIST Engineering Laboratory.

The National Institute of Standards and Technology is an agency of the U.S. Department of Commerce. 